\title{
18. CENOZOIC ASH LAYERS ON THE VØRING PLATEAU (ODP LEG 104)1
}

\author{
P. R. Bitschene, H.-U. Schmincke, and L. Viereck ${ }^{2}$
}

\begin{abstract}
Numerous fresh ash layers comprise about $0.3 \%$ by volume of Neogene to Holocene sediments drilled at Leg 104 Sites 642 and 643 (Vøring Plateau, North Atlantic). Median grain sizes of the ashes are about $100 \mu \mathrm{m}$ and maximum grain sizes range up to $1200 \mu \mathrm{m}$. Rhyolitic pumice shards dominate, with minor bubble wall shards. Basaltic shards are poorly vesicular and blocky or round. Phenocrystic plagioclase, zircon, and clinopyroxene occur in the rhyolitic, plagioclase, and clinopyroxene phenocrysts and basaltic lithics in the basaltic tephra. Quartz, amphibole, clinozoisite, and rutile are interpreted as xenocrysts. All ash layers are well-sorted and represent distal fallout from major explosive eruptions. Most ashes are rhyolitic (high-K and low-K) in composition, some are bimodal (tholeiitic and rhyolitic). Early Miocene tephra is dominantly basaltic.

Iceland is inferred to be the likely source region for most ashes. Late Miocene high-K rhyolites may have originated from the K-rich Jan Mayen magmatic province. One Quaternary layer with biotite and alkali feldspar phenocrysts may have been derived from Jan Mayen Island. Four individual Pliocene to Holocene ash layers from Sites 642 and 643 can be correlated fairly well. Upper Miocene layers are tentatively correlated as a sequence between Sites 642 and 643 .

Average calculated layer frequencies are about three layers/m.y. through the Pliocene and Pleistocene and five to eight layers per m.y. through the middle and late Miocene, suggesting rather continuous volcanic activity in the North Atlantic. Episodic magmatic activity during Neogene epochs in this part of the North Atlantic, as postulated in the literature, cannot be confirmed.
\end{abstract}

\section{INTRODUCTION}

Numerous ash layers encountered in the sedimentary sequence of the Vøring Plateau, northeast Atlantic Ocean, drilled during Leg 104 at Sites 642, 643, and 644 (Fig. 1), provide a unique record of Cenozoic explosive volcanism in the North Atlantic (Eldholm, Thiede, Taylor, et al., 1987). A basement high, upon which Site 642 is located with a northeast-trending linear escarpment, divides the Vøring Plateau into the inner plateau to the east and the outer plateau to the west (Fig. 1). Site 643 is located at the base of the western slope of the outer Vøring Plateau, and Site 644 lies within the inner Vøring Plateau. Ash layers are not abundant in Pliocene to Holocene sediments from the latter site and were not studied in detail.

Fifty-four distinct ash layers were identified within Holes 642B, 642C, and 642D. Hole 643A contained 56 distinct ash layers; only five ash layers were recovered in the Pliocene to Holocene section of Site 644 (Eldholm, Thiede, Taylor, et al., 1987).

Excellent core recovery $(79 \%-98 \%)$ and freshness of the glass made a study of distinct tephra layers promising. The aim of our study is to help unravel the stratigraphy of Sites 642 and 643 by using geochemical and mineralogical compositions of prominent tephra layers. Also, an attempt is made to infer potential source regions for the ash, and to draw some conclusions regarding possible episodic volcanic activity during the Neogene and Quaternary.

\section{METHODS}

Based on shipboard descriptions, 16 fresh and representative samples were selected for detailed study. The ash layers studied are listed in Figure 4. Grain size and sorting of glass shards, morphology, color, and crystal and lithic components and their abundances were studied in

\footnotetext{
${ }^{1}$ Eldholm, O., Thiede, J., Taylor, E., et al., 1989. Proc. ODP, Sci. Results, 104: College Station, TX (Ocean Drilling Program).

2 Institut für Mineralogie, Ruhr-Universität, Postfach 102148, D-4630 Bochum, Federal Republic of Germany.
}

smear slides and thin sections. Between 20 and 50 grains in each thin section were measured using calibrated ocular scales.

Major-element compositions of individual glass shards and mineral grains were determined with a CAMEBAX automated electron microprobe using natural mineral and glass standards. Raw data were corrected using the program CORREX (CAMEBAX). Operation conditions: Accelerating voltage $15 \mathrm{KV}$, beam spot size $5 \times 5 \mu \mathrm{m}$ and beam current of $14 \mathrm{nA}$. $\mathrm{Na}_{2} \mathrm{O}$ concentrations measured are lower by ca. $1 \%-2 \%$ by weight, when compared with compositions of rhyolitic shards and rocks from the North Atlantic and Iceland (Table 1). This could be due to selective sodium loss during diagenesis or, more likely, during microprobe analysis. $\mathrm{K}_{2} \mathrm{O}$ concentrations are slightly higher than in rocks of similar bulk compositions from Iceland (Walker, 1966; Jorgensen, 1980; Schmincke et al., 1982).

\section{RESULTS}

\section{Stratigraphy and Lithology}

The ash layers comprise about $0.3 \%$ of the sediments and occur within Neogene and Quaternary sediments of the Vøring Plateau (Fig. 2). They represent the most complete succession of Cenozoic ash layers so far recovered in the North Atlantic. At Site 642, the Neogene sequence rests upon an Eocene massive, glauconite-rich, volcaniclastic mass flow deposit with an erosional unconformity (Fig. 2). At Site 643, the Oligocene to Holocene section overlies an Eocene volcaniclastic sequence with a basal conglomerate unit consisting of reworked basalt.

Tephra occurs as discrete (sharp base and gradational top; $L$ in Fig. 4) or disseminated (no sharp base and up to $80 \%$ ooze or fossiliferous mud; $D$ in Fig. 4 ) ash layers. Enriched layers ( $E$ in Fig. 4) often overlie discrete or disseminated layers by as much as $100 \mathrm{~cm}$ and contain only $5 \%-10 \%$ ash. Ash pods are distributed throughout the cores. A complete list of all ash layers and their corresponding raw data is given in Eldholm, Thiede, Taylor, et al. (1987).

Most ash layers are Miocene in age, and are particularly abundant between 113 and 137 meters below sea floor (mbsf) at Site 642 and 90 and 110 mbsf at Site 643 . Only 12 layers are preserved within the uppermost $110 \mathrm{~m}$ of Site 642 , and 11 layers are 


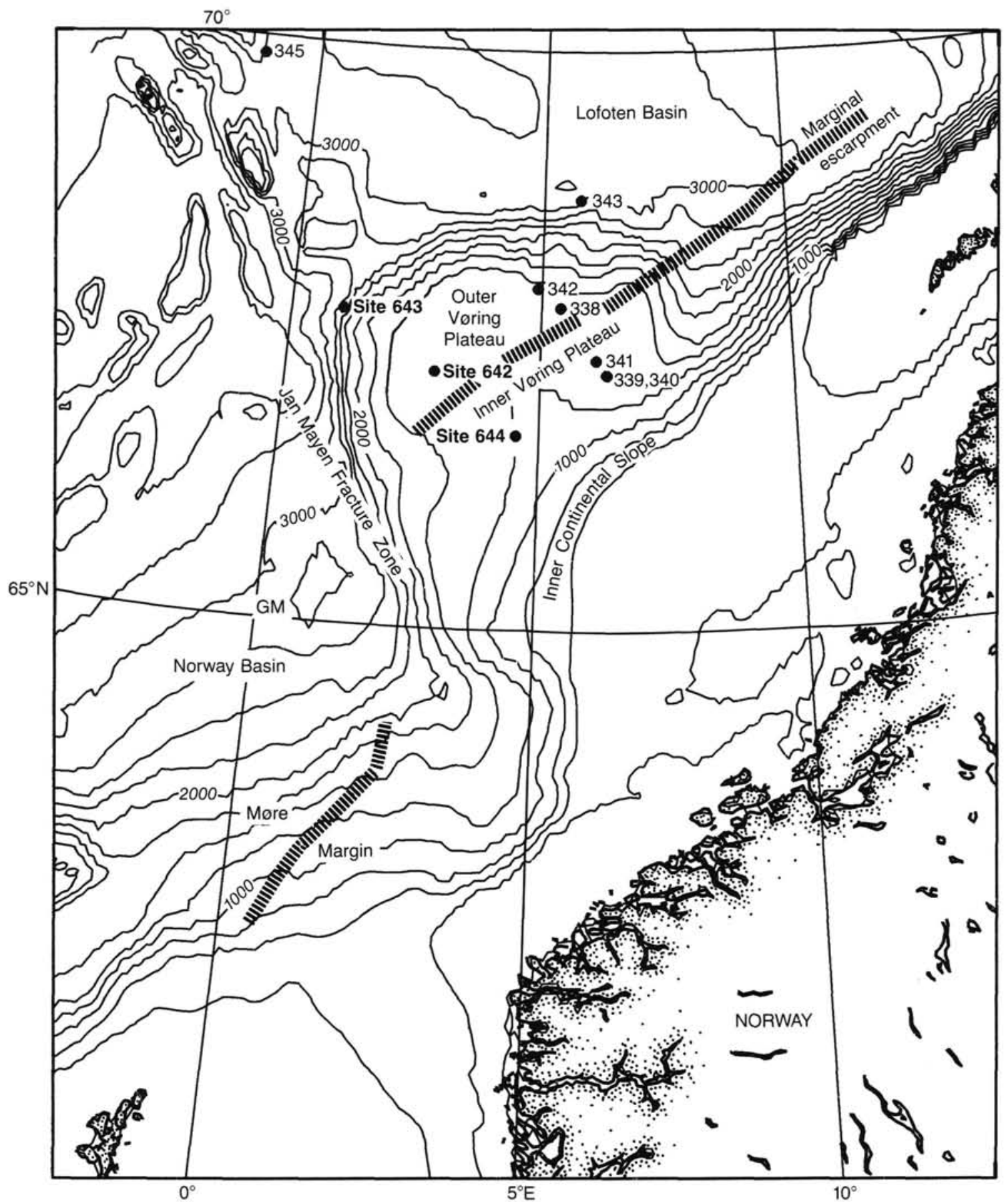

Figure 1. Bathymetry of the Norwegian continental margin $60^{\circ}-70^{\circ} \mathrm{N}$ (contour interval, $250 \mathrm{~m}$ ). Distance between Sites 642 and 643 is about $70 \mathrm{~km}$.

preserved within the uppermost $90 \mathrm{~m}$ at Site 643 , representing Pliocene to Holocene volcanic activity.

Tephra layers from Site 642 are mostly between 2 and $4 \mathrm{~cm}$ thick, but range from less than 1 to as much as $11 \mathrm{~cm}$ in thickness (Fig. 3). Site 643 ash layers are, on the average, thinner than those at Site 642 (Fig. 3); one layer, however, is $11 \mathrm{~cm}$ thick. Color and thus inferred chemical composition do not correlate with ash layer thickness.

All ash layers consist dominantly of vitric ash with few crystals and lithic clasts. Many are white to gray, but can be dark gray to black when surface pyritized (e.g., interval 104-642D-7-
$6,62-74 \mathrm{~cm})$. They are well-sorted with an average median grain size of approximately $100 \pm 40 \mu \mathrm{m}$ (fine sands to coarse silts). Maximum grain sizes ( 3 largest shards), however, differ significantly and range from about $240 \mu \mathrm{m}$ to as much as $1200 \mu \mathrm{m}$ (Section 104-642B-13, CC, 9-13 cm). Colorless shards are larger than brown glass clasts in the same layer. Maximum grain size is grossly related to the colorless shards (Fig. 4).

Different shard types can be distinguished by color, refractive index (R. I.), and dominant shape: colorless to light-greenish shards with an R. I. $<1.54$ consist of angular tubular pumice and minor platy and cuspate shards (vesicle walls and vesicle 
Table 1. Compilation of chemical data on North Atlantic rhyolitic ashes (Sigurdsson and Loebner, 1981), first three columns, and Iceland rhyolitic rocks (middle three columns), in comparison with Leg 104 rhyolitic ash.

\begin{tabular}{|c|c|c|c|c|c|c|c|c|c|c|}
\hline & Low $-\mathrm{K}$ & High-K & High-K & $\begin{array}{l}\text { Walker } \\
(1966)\end{array}$ & Schmincke & $\begin{array}{l}\text { et al } \\
\text { (1982) }\end{array}$ & $\begin{array}{r}\text { Jorgensen } \\
(1980)\end{array}$ & $\begin{array}{c}642-B-13-C C, 9-13 \\
(O D P, \text { LEG 104) }\end{array}$ & $\begin{array}{r}642-C-15-2,110-114 \\
\text { (ODP, Leg 104) }\end{array}$ & $\begin{array}{l}643-11-1,104-105 \\
\text { (ODP, Leg 104) }\end{array}$ \\
\hline $\begin{array}{r}\mathrm{SiO2} \\
\mathrm{T} 102 \\
\mathrm{~A} 1203 \\
\mathrm{FeO} \\
\mathrm{MgO} \\
\mathrm{CaO} \\
\mathrm{Na2O} \\
\mathrm{K} 2 \mathrm{O}\end{array}$ & $\begin{array}{r}72,74 \\
0,13 \\
11,94 \\
2,74 \\
0,04 \\
1,16 \\
5,02 \\
2,81\end{array}$ & $\begin{array}{r}72,57 \\
0,17 \\
13,33 \\
1,70 \\
0,10 \\
0,77 \\
4,49 \\
4,33\end{array}$ & $\begin{array}{r}73,61 \\
0,20 \\
9,46 \\
4,45 \\
0,00 \\
0,13 \\
4,67 \\
4,58\end{array}$ & $\begin{array}{r}73,50 \\
0,31 \\
13,30 \\
3,21 \\
0,33 \\
1,64 \\
4,46 \\
3,03\end{array}$ & & $\begin{array}{r}72,60 \\
0,33 \\
13,60 \\
3,62 \\
0,28 \\
0,84 \\
4,97 \\
3,41\end{array}$ & $\begin{array}{r}73,60 \\
0,28 \\
12,50 \\
3,01 \\
0,16 \\
0,98 \\
5,13 \\
4,09\end{array}$ & $\begin{array}{r}72,53 \\
0,20 \\
13,02 \\
1,72 \\
0,13 \\
0,79 \\
2,95 \\
4,09\end{array}$ & $\begin{array}{r}73,42 \\
0,06 \\
12,56 \\
1,26 \\
0,04 \\
0,53 \\
3,29 \\
4,28\end{array}$ & $\begin{array}{r}74,56 \\
0,20 \\
11,53 \\
2,32 \\
0,08 \\
0,95 \\
3,06 \\
2,69\end{array}$ \\
\hline sum & 96,58 & 97,46 & 97,10 & 99,78 & & 99,65 & 99,75 & 95,43 & 95,44 & 95,39 \\
\hline
\end{tabular}

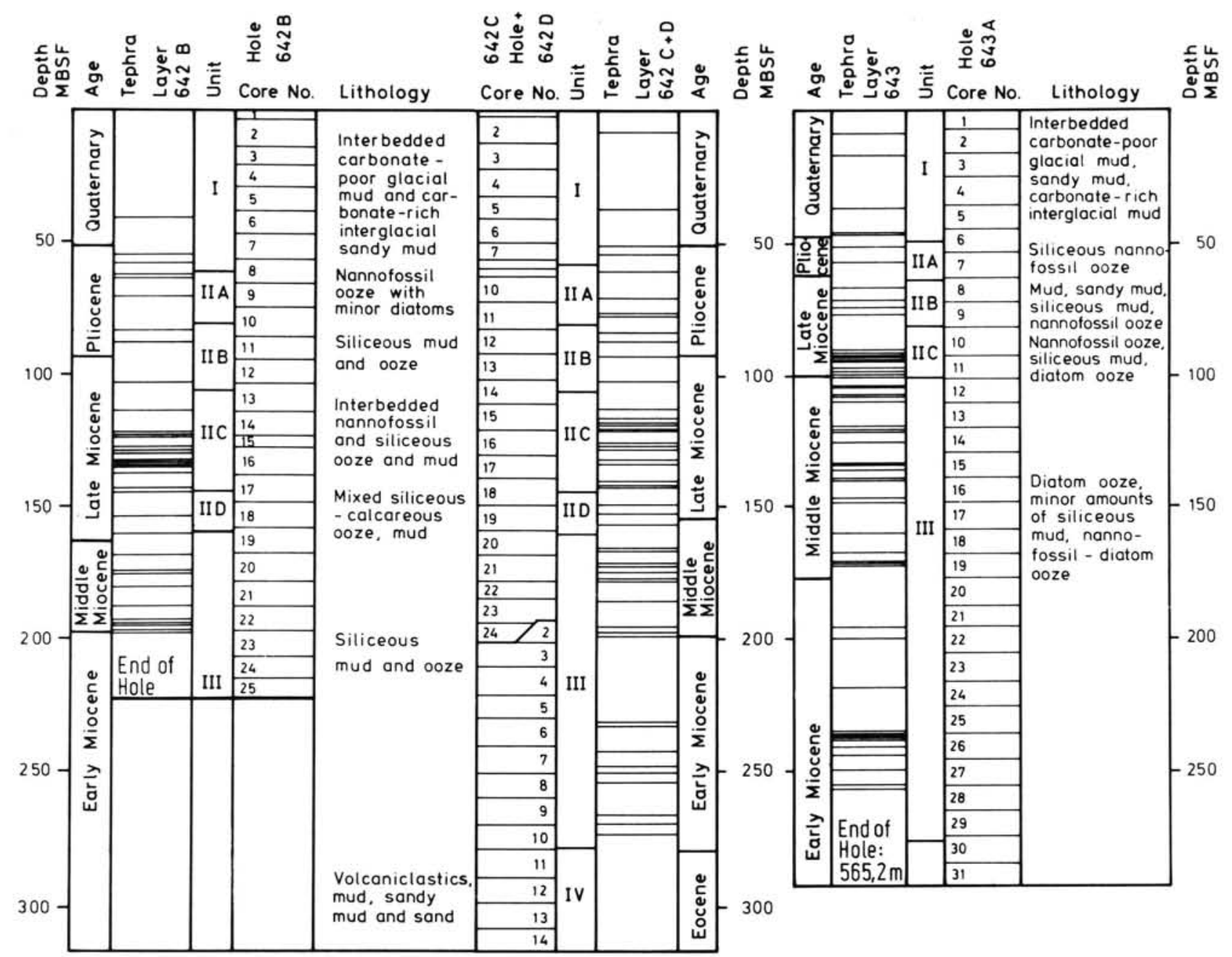

Figure 2. Stratigraphic position of tephra layers in Holes 642B, 642C, 642D, and 643A (data from Eldholm, Thiede, Taylor, et al., 1987; new stratigraphic summary after Goll, this volume).

junctions); light-brown shards with R. I. > 1.54 consist of tubular pumice and blocky, platy, and cuspate shards; deep-brown shards with R. I. > 1.54 have round (droplets) and blocky, platy shapes and few but round vesicles. Additional glass phases consist of altered, ore-spotted and/or hydrated glasses and opaque tachylite. Shipboard studies of color and R. I. suggest the following magma types: rhyolite (colorless glass), icelandite (light brownish glass), and basaltic to andesitic varieties (brown glass). However, brown shards with low R. I. in rhyolitic tephra layers are also rhyolitic in composition based on microprobe data (Table 2).

Colorless glass populations show up to $80 \%$ (Sample 104$642 \mathrm{C}-15-2,111-112 \mathrm{~cm}$ ) angular, tubular pumices with parallel alignment of elongate vesicles. The remaining $20 \%$ are made up of platy and cuspate shards with smaller grain size and lack of bubbles. Light-brown glass mostly occurs as fibrous tubular pumice and minor blocky, vesicle-free shards. Deep-brown shards are blocky, platy to cuneiform, often round droplets with spheri- 


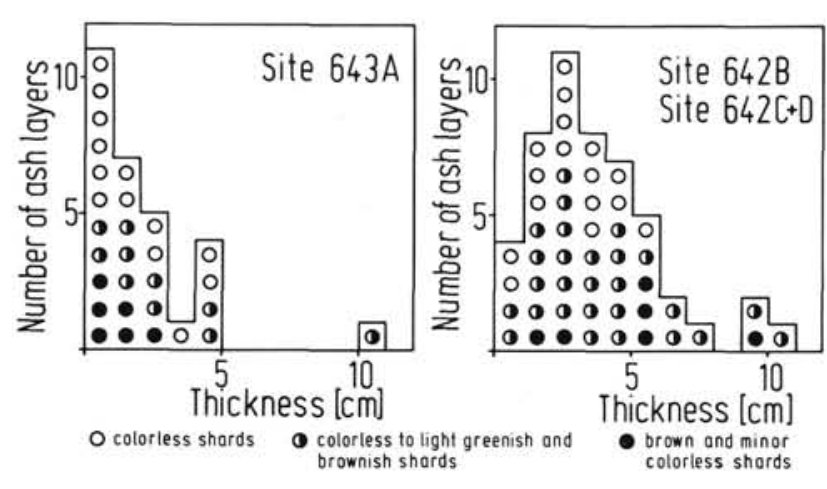

Figure 3. Histogram of thicknesses of discrete and disseminated ash layers and distribution of their colorless and brown shards.

cal vesicles. Some brown shards contain clinopyroxene and plagioclase phenocrysts, others are spotted or rimmed with sulfides.

Layers of brown, basaltic glass shards are dominant in the lower part of the sedimentary section, which was only recovered in Holes 642D and 643 (Fig. 4). There is, however, always a significant fraction of brown, not necessarily basaltic, glass in ash layers that consist mostly of colorless glass (Fig. 4). A unique occurrence of crackled glass occurs in layer 104-642D-7-6, 70$74 \mathrm{~cm}$. The brown shards, up to $300 \mu \mathrm{m}$ in diameter, are broken into polygonal domains. Some plagioclase phenocrysts are also cracked. Alteration of glass (incipient palagonitization) is evident along the microcracks.

\section{Mineral Assemblages}

Crystals and lithics are minor constituents ( $<2 \%$ by vol.). Layers 104-642B-6-1, 131-132 cm, 104-643A-7-3, 66-67 cm, $104-643 \mathrm{~A}-15-4,147-149 \mathrm{~cm}$, and 104-643A-19-4, 116-117 cm are, however, significantly enriched in crystals and lithics. Most crystal diameters are less than $120 \mu \mathrm{m}$, although biotite, and a few zircon and quartz fragments are up to $180 \mu \mathrm{m}$ long.

Minerals in an ash layer (Fig. 4) can be either phenocrysts or xenocrysts derived from wall rock during the eruptive process. Tephra horizons with dominantly brown, basaltic shards (intervals 104-642D-11-1, 83-84 cm, and 104-642D-7-6, 70-74 cm) contain plagioclase, clinopyroxene, and opaque minerals with appreciable detrital quartz. Tephra with colorless, rhyolitic glass (intervals 104-642C-15-2, 86-87 cm, and 104-643A-11-1, 104$105 \mathrm{~cm}$ ) contains plagioclase, clinopyroxene, and zircon phenocrysts with some quartz xenocrysts. Biotite is the major phenocryst phase in layer 104-642B-6-1, 131-132 cm. Some amphibole, clinozoisite, quartz, and feldspar is probably derived from the wall rock or detrital (intervals 104-643A-15-4, 147-149 cm, and 104-643A-19-4, 116-117 cm). Opaques (oxides, sulfides, hydroxides, tachylites) and carbonate and typical diagenetic minerals (glauconite, sheet silicates, zeolites?) are additional components.

Anhedral quartz grains, the most abundant crystal components, show large variations in grain size and contain tiny inclusions. Most, if not all, of the quartz grains in the disseminated ash layers are thought to be detrital. Plagioclase is always twinned, shows some euhedral laths within brown basaltic glass, and occurs as anhedral splinters in the rhyolitic ash layers. Freshness and coexistence of plagioclase and some clinopyroxene with brown glass suggest these minerals to be phenocrystic. K-feldspar is a minor, simply-twinned, constituent. Phenocrystic zircon is faintly gray to clear and some grains show single crystal faces. Very rare rutile is brownish orange, elongate, and proba- bly detrital. Clinopyroxene is light greenish gray in brown glass and shows rare twinning and cleavage. Blue to greenish pleochroic amphiboles (alkali-amphiboles) and brownish pleochroic amphiboles are very rare.

Lithics, composed of clusters of glomerophyric plagioclase in a devitrified matrix, occur in layer 104-643A-15-4, 147-149 $\mathrm{cm}$. Unique mineral assemblages cannot be assigned to specific layers except for the biotite-rich layer 104-642B-6-1, 131-132 cm (Fig. 4).

\section{Geochemistry}

One prominent upper Miocene rhyolitic ash layer from each hole, three in total (104-642B-13-CC, 9-13 cm; 104-642C-15-2, $110-114 \mathrm{~cm}$; and 104-643A-11-1, 104-105 cm), was selected for chemical analysis. Based on shipboard interpretation (Eldholm, Thiede, Taylor, et al., 1987), the layers are thought to correlate well between Holes $643 \mathrm{~A}$ and $642 \mathrm{~B}$ and $642 \mathrm{C} / 642 \mathrm{D}$, the distance being only $450 \mathrm{~m}$ between the Holes 642B and 642C/ 642D. Compositions of plagioclase were also determined.

All shards analyzed from these layers, including brown shards, are rhyolitic in composition (Table 2, Fig. 5). The brown color of some rhyolitic shards is probably due to the oxidation of iron. Each analyzed layer has a characteristic composition significantly different from the other two layers in their $\mathrm{SiO}_{2}, \mathrm{Al}_{2} \mathrm{O}_{3}$, $\mathrm{FeO}$, and $\mathrm{CaO}$ concentrations (Table 2, Fig. 5). Glasses from layers 104-642B-13-CC, 9-13 cm and 104-642C-15-2, 110-114 $\mathrm{cm}$ are $\mathrm{K}$-rich alkali rhyolites with $\mathrm{K}_{2} \mathrm{O}$ concentrations $>3.8 \%$ by weight. In contrast, layer $104-643 \mathrm{~A}-11-1,104-105 \mathrm{~cm}$ is low in $\mathrm{K}_{2} \mathrm{O}(<2.9 \mathrm{wt} \%)$, except for a single grain, but higher in $\mathrm{FeO}$ and $\mathrm{CaO}$ concentrations (Table 2, Fig. 5).

The more calcic plagioclase (andesine) is found in the more K-rich magma (Table 3), whereas the more sodic plagioclase (oligoclase) is found in the $\mathrm{K}$-poor magma.

The basaltic shards from layer 104-642C-10-6, 5-7 cm, exhibit high $\mathrm{MgO}(6.07-6.63 \mathrm{wt} \%), \mathrm{CaO}(11.97-12.50 \mathrm{wt} \%)$, and FeO (12.62-13.45 wt\%), intermediate $\mathrm{TiO}_{2}(2.39-2.66 \mathrm{wt} \%)$, and low $\mathrm{SiO}_{2}(48.20-48.54 \mathrm{wt} \%)$, and $\mathrm{K}_{2} \mathrm{O}(0.27-0.34 \mathrm{wt} \%)$ concentrations. The shards are uniformly tholeiitic (medium-Ti basalts) and differ from the more evolved tholeiites (high-Ti basalts) from layer 104-643A-7-3, 66-67 cm (Fig. 6), which are lower in $\mathrm{MgO}(4.63-5.19 \mathrm{wt} \%)$ and $\mathrm{CaO}(9.70-10.03 \mathrm{wt} \%)$ but distinctly higher particularly in $\mathrm{TiO}_{2}(3.39-4.21 \mathrm{wt} \%), \mathrm{FeO}(13.90$ $15.37 \mathrm{wt} \%), \mathrm{K}_{2} \mathrm{O}(0.60-0.72 \mathrm{wt} \%)$ and $\mathrm{P}_{2} \mathrm{O}_{5}(0.37-0.51 \mathrm{wt} \%)$. The more evolved basaltic glasses from layer 104-643A-2-3, 74$75 \mathrm{~cm}$, have $\mathrm{SiO}_{2}$ concentrations between 48.62 and $50.13 \mathrm{wt} \%$ and $\mathrm{MgO}$ concentrations between 4.10 and $4.34 \mathrm{wt} \%$. They are high-Ti basalts (3.23-3.43 wt \% $\left.\mathrm{TiO}_{2}\right)$ with high $\mathrm{FeO}(13.06-$ $14.03 \mathrm{wt} \%)$ and $\mathrm{K}_{2} \mathrm{O}(0.75-0.83 \mathrm{wt} \%)$ concentrations (Fig. 6, Table 2). The major element concentrations of the basaltic shards analyzed are similar to the composition of basalts from Iceland (e.g., Schmincke et al., 1982; Flower et al., 1982) and can be divided into more primitive, medium-Ti basalts and more evolved high- $\mathrm{Ti}$ basalts with markedly higher $\mathrm{FeO}$ and $\mathrm{K}_{2} \mathrm{O}$ concentrations.

The $\mathrm{SiO}_{2}$-rich (73.69-76.90 wt\%) rhyolitic shards found in two layers (Table 2) are enriched in FeO (2.44-3.60 wt \%) and $\mathrm{CaO}(1.07-2.13 \mathrm{wt} \%)$. Preliminary analyses of some rhyolitic shards from layer $104-643 \mathrm{~A}-2-3,74-75 \mathrm{~cm}$ show also high $\mathrm{CaO}$ (1.24-1.45 wt \%) and $\mathrm{FeO}(2.74-3.16 \mathrm{wt} \%)$ concentrations. The major-element compositions of the rhyolitic shards from these layers clearly differ from those of rhyolitic shards from purely rhyolitic ash layers 104-642B-13CC, 9-13 cm, 104-642C-15-2, $110-114 \mathrm{~cm}$, and 104-643A-11-1, 104-105 cm (Table 2). More data, however, are needed to decide whether rhyolite shards from bimodal ash deposits differ systematically from those of purely rhyolitic ash layers or not. 


\begin{tabular}{|c|c|c|c|c|c|c|c|c|}
\hline$\frac{0}{1}$ & Sample & 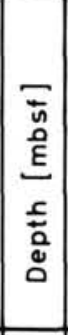 & 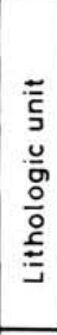 & 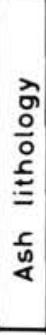 & 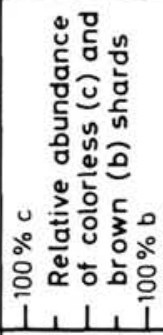 & 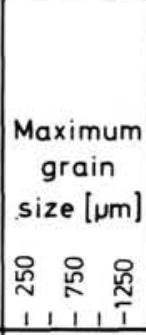 & 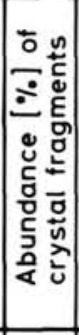 & Mineral assemblages \\
\hline \begin{tabular}{l}
$\infty$ \\
\multirow{2}{*}{}
\end{tabular} & $\begin{array}{l}6-1-131-132 \\
9-3-95-96 \\
9-3-106-107 \\
13 \mathrm{CC}-10-12\end{array}$ & $\begin{array}{r}41 \\
71 \\
71 \\
114\end{array}$ & $\begin{array}{l}\text { I } \\
\text { II A } \\
\text { II A } \\
\text { II C }\end{array}$ & $\begin{array}{l}L \\
L \\
E \\
L\end{array}$ & $\bullet$ & $\begin{array}{cc}x & \\
x & \\
x & \\
& x\end{array}$ & $\begin{array}{l}- \\
0 \\
0 \\
0\end{array}$ & $\begin{array}{l}\text { B, Kt, Cpx, Zr, Op, Q, PI } \\
\text { Q, PI, Cpx, Cz, Zr, Op, Ca } \\
\text { Q, PI, Cpx, Op, Ca, Ru? } \\
\text { O, PI, Cpx, Op, Zr, Kf? }\end{array}$ \\
\hline 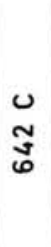 & $\begin{array}{l}10-6-5-7 \\
15-2-86-87 \\
15-2-111-112 \\
15-4-88-93 \\
19-3-71-74\end{array}$ & $\begin{array}{l}71 \\
114 \\
114 \\
117 \\
153\end{array}$ & $\begin{array}{l}\text { IIA } \\
\text { II C } \\
\text { IIC } \\
\text { IIC } \\
\text { IID }\end{array}$ & $\begin{array}{l}L \\
L \\
D \\
L \\
L\end{array}$ & $\begin{array}{l}\bullet \\
\bullet \\
\bullet \\
\bullet\end{array}$ & $\begin{array}{r}x \\
x \\
x \\
x\end{array}$ & $\begin{array}{l}0 \\
0 \\
0 \\
0 \\
0\end{array}$ & $\begin{array}{l}\text { Q, PI, Cpx, Op, B? } \\
\text { Q, PI, Zr, Op, Cpx, Kf? } \\
\text { Q, PI, Zr, Op, Cpx? } \\
\text { Q, PI, Cpx, Zr, Op, Kf, Am? } \\
\text { Q, PI, Cpx }\end{array}$ \\
\hline กิ & $\begin{array}{c}7-6-70-74 \\
11-1-83-84\end{array}$ & $\begin{array}{l}247 \\
278\end{array}$ & $\begin{array}{l}\text { III } \\
\text { III }\end{array}$ & $\begin{array}{l}P \\
L\end{array}$ & • & $\begin{array}{l}x \\
x\end{array}$ & $\begin{array}{l}0 \\
0\end{array}$ & $\begin{array}{l}\mathrm{PI}, \mathrm{Cpx}, \mathrm{Q}, \mathrm{Op}, \mathrm{GI} \\
\text { crackled + palagonit. glass } \\
\mathrm{PI}, \mathrm{Cpx}, \mathrm{Q}, \mathrm{Kf} \text { ?, Am? }\end{array}$ \\
\hline ঙุ. & $\begin{array}{l}2-3-74-75 \\
7-3-66-67 \\
11-1-104-105 \\
15-4-147-149 \\
19-4-116-117\end{array}$ & $\begin{array}{r}8 \\
57 \\
93 \\
136 \\
173\end{array}$ & $\begin{array}{l}\text { I } \\
\text { IIA } \\
\text { IIC } \\
\text { III } \\
\text { III }\end{array}$ & $\begin{array}{l}L \\
P \\
L \\
L \\
L\end{array}$ & $\begin{array}{l}\bullet \\
\bullet \\
\bullet \\
\bullet\end{array}$ & $\begin{array}{c}x \\
\times \\
x \\
x\end{array}$ & $\begin{array}{l}0 \\
0 \\
0 \\
0 \\
0\end{array}$ & $\begin{array}{l}\text { Q, PI, Cpx, Zr, Op } \\
\text { Q, PI, Cpx } \\
\text { Q, PI, Cpx, Zr, Op } \\
\text { Q, PI, Cpx, Zr, Cz, Op } \\
\text { Q, PI, Cpx, Am? Op, Zr }\end{array}$ \\
\hline
\end{tabular}

$$
\text { Note: } \begin{aligned}
& L=\text { Discrete ash layer } \\
& E=\text { Ash }- \text { rich layer } \\
& D=\text { Disseminated ash } \\
& P=\text { Ash pods }
\end{aligned}
$$

$$
\begin{aligned}
& B=\text { Biotite } \\
& \mathrm{Pl}=\text { Plagioclase } \\
& \mathrm{Kf}=\mathrm{K} \text { - Feldspar } \\
& Q=\text { Quartz } \\
& \mathrm{Cpx}=\text { Clinopyroxene } \\
& \mathrm{Am}=\text { Amphibole } \\
& \mathrm{Zr}=\text { Zircon } \\
& \mathrm{Ca}=\text { Calcite } \\
& \mathrm{C}_{2}=\text { Clinozoisite } \\
& \mathrm{OP}_{\mathrm{P}}=\text { Opaques and tachylite } \\
& \mathrm{Gl}=\text { Glauconite }
\end{aligned}
$$$$
\text { o rare, }<2 \%
$$$$
\text { - common, } 2-5 \%
$$$$
\text { - abundant, > } 5 \%
$$

Figure 4. Petrographic description and position of prominent tephra layers.

\section{DISCUSSION}

Neogene sediments from the Vøring Plateau in the North Atlantic contain a unique record of interbedded ash layers. The ash layers investigated from Sites 642 and 643 of Leg 104 have similar median grain sizes and mineral assemblages but differ in the composition of the glass (basaltic and rhyolitic, some bimodal compositions).

\section{Eruption, Transport, and Sedimentation}

Most glass clasts are colorless pumice and bubble wall shards. The light-brown shards are also highly vesicular pumices with elongate vesicles; deep-brown shards are mostly bubble-free, blocky, and platy shards. The vitric ashes are interpreted as distal fallout products of powerful Plinian eruptions because of the well-sorted character of all ash samples, although a few large grains possibly indicate minor contributions from pumice rafting.

Brown, basaltic glasses are common in lower to middle Miocene ash layers, which consist of brown, blocky shards and round droplets. Crackled, bubble-free glass and phenocrysts in layer 104-642D-7-6, 70-74 cm, are most likely caused by shockcooling in contact with cold water (or ice?) and subsequent cracking (Fisher and Schmincke, 1984). These ash layers are in- 
Table 2. Microprobe analyses of glass shards from Holes 642B, 642C, and 643A (total Fe as FeO).

\begin{tabular}{|c|c|c|c|c|c|c|c|c|c|c|c|}
\hline Sample & $\mathrm{SiO} 2$ & $\mathrm{TiO} 2$ & A1203 & $\mathrm{FeO}$ & $\mathrm{MnO}$ & Mgo & $\mathrm{CaO}$ & $\mathrm{Na} 2 \mathrm{O}$ & K2O & P205 & Sum \\
\hline $642 B-13-C C, 9-13$ & $\begin{array}{l}72,52 \\
71,93 \\
72,49 \\
72,11 \\
72,34 \\
72,86 \\
72,12 \\
72,48 \\
73,01 \\
72,46 \\
72,99 \\
73,04\end{array}$ & $\begin{array}{l}0.22 \\
0.22 \\
0.20 \\
0.20 \\
0.17 \\
0.19 \\
0.21 \\
0.18 \\
0.23 \\
0.14 \\
0.20 \\
0,22\end{array}$ & $\begin{array}{l}13,04 \\
13,20 \\
12,98 \\
12,95 \\
12,98 \\
13,01 \\
13,03 \\
13,11 \\
12,83 \\
13,08 \\
13,16 \\
12,88\end{array}$ & $\begin{array}{l}1.68 \\
1.75 \\
1.80 \\
1.55 \\
1.65 \\
1.71 \\
1.76 \\
1.73 \\
1.80 \\
1.65 \\
1.84 \\
1.71\end{array}$ & $\begin{array}{l}0.04 \\
0.07 \\
0.03 \\
0.08 \\
0.08 \\
0.09 \\
0.05 \\
0.08 \\
0.05 \\
0.01 \\
0.03 \\
0.05\end{array}$ & $\begin{array}{l}0.12 \\
0.15 \\
0.13 \\
0.14 \\
0,13 \\
0.11 \\
0.14 \\
0.12 \\
0.13 \\
0.15 \\
0.11 \\
0.15\end{array}$ & $\begin{array}{l}0.86 \\
0.73 \\
0.81 \\
0.80 \\
0.77 \\
0.75 \\
0.78 \\
0.75 \\
0.80 \\
0.79 \\
0.81 \\
0.83\end{array}$ & $\begin{array}{l}3.09 \\
3.09 \\
2.68 \\
2.97 \\
3.07 \\
3.06 \\
2.85 \\
3.08 \\
2.72 \\
3.05 \\
3.06 \\
2.69\end{array}$ & $\begin{array}{l}3.95 \\
4.20 \\
4.01 \\
4.58 \\
4.08 \\
4.16 \\
4.28 \\
3.86 \\
3.92 \\
3.83 \\
4.24 \\
3.93\end{array}$ & $\begin{array}{l}0.10 \\
0.03 \\
0.04 \\
0.06 \\
0.00 \\
0.00 \\
0.00 \\
0.01 \\
0.02 \\
0.05 \\
0.01 \\
0.01\end{array}$ & $\begin{array}{l}95,62 \\
95,37 \\
95,17 \\
95,44 \\
95,27 \\
95,94 \\
95,22 \\
95,40 \\
95,51 \\
95,21 \\
96,45 \\
95,51\end{array}$ \\
\hline $\begin{array}{l}\text { Average } \\
\text { Minimum } \\
\text { Maximum }\end{array}$ & $\begin{array}{l}72,53 \\
71,93 \\
73,04\end{array}$ & $\begin{array}{l}0.20 \\
0.14 \\
0.23\end{array}$ & $\begin{array}{l}13,02 \\
12,83 \\
13,20\end{array}$ & $\begin{array}{l}1.72 \\
1.55 \\
1.84\end{array}$ & $\begin{array}{l}0.06 \\
0.01 \\
0.09\end{array}$ & $\begin{array}{l}0,13 \\
0.11 \\
0.15\end{array}$ & $\begin{array}{l}0.79 \\
0.73 \\
0.86\end{array}$ & $\begin{array}{l}2.95 \\
2,68 \\
3.09\end{array}$ & $\begin{array}{l}4,09 \\
3,83 \\
4,58\end{array}$ & $\begin{array}{l}0,03 \\
0,00 \\
0,10\end{array}$ & $\begin{array}{l}95,51 \\
95,17 \\
96,45\end{array}$ \\
\hline $642 C-15-2, \quad 110-114$ & $\begin{array}{l}73,28 \\
73,20 \\
73,47 \\
73,56 \\
73,41 \\
73,45 \\
73,63 \\
73,35\end{array}$ & $\begin{array}{l}0,06 \\
0,04 \\
0,07 \\
0,10 \\
0,01 \\
0,07 \\
0,06 \\
0,06\end{array}$ & $\begin{array}{l}12,46 \\
12,51 \\
12,52 \\
12,61 \\
12,50 \\
12,76 \\
12,69 \\
12,44\end{array}$ & $\begin{array}{l}1,36 \\
1,26 \\
1,13 \\
1,28 \\
1,30 \\
1,25 \\
1,27 \\
1,26\end{array}$ & $\begin{array}{l}0.07 \\
0.07 \\
0.01 \\
0.08 \\
0.06 \\
0.02 \\
0.05 \\
0.03\end{array}$ & $\begin{array}{l}0.04 \\
0.02 \\
0.04 \\
0.04 \\
0.04 \\
0.04 \\
0.04 \\
0.03\end{array}$ & $\begin{array}{l}0,55 \\
0,51 \\
0,50 \\
0,50 \\
0,52 \\
0,55 \\
0,52 \\
0,58\end{array}$ & $\begin{array}{l}3,55 \\
3,44 \\
3,07 \\
3,07 \\
3,08 \\
3,21 \\
3,51 \\
3,39\end{array}$ & $\begin{array}{l}4,60 \\
4,29 \\
4,57 \\
4,24 \\
4,25 \\
4,05 \\
4,19 \\
4,01\end{array}$ & $\begin{array}{l}0,01 \\
0,01 \\
0.02 \\
0.02 \\
0.24 \\
0,03 \\
0.01 \\
0.01\end{array}$ & $\begin{array}{l}95,98 \\
95,35 \\
95,40 \\
95,50 \\
95,41 \\
95,43 \\
95,97 \\
95,16\end{array}$ \\
\hline $\begin{array}{l}\text { Average } \\
\text { Minimum } \\
\text { Maximum }\end{array}$ & $\begin{array}{l}73,42 \\
73,20 \\
73,63\end{array}$ & $\begin{array}{l}0,06 \\
0,01 \\
0,10\end{array}$ & $\begin{array}{l}12,56 \\
12,44 \\
12,76\end{array}$ & $\begin{array}{l}1,26 \\
1.13 \\
1,36\end{array}$ & $\begin{array}{l}0.05 \\
0.01 \\
0.08\end{array}$ & $\begin{array}{l}0.04 \\
0.02 \\
0.04\end{array}$ & $\begin{array}{l}0.53 \\
0.50 \\
0.58\end{array}$ & $\begin{array}{l}3.29 \\
3.07 \\
3.55\end{array}$ & $\begin{array}{l}4,28 \\
4,01 \\
4,60\end{array}$ & $\begin{array}{l}0.04 \\
0.01 \\
0.24\end{array}$ & $\begin{array}{l}95,52 \\
95,16 \\
95,98\end{array}$ \\
\hline $643 A-11-1, \quad 104-105$ & $\begin{array}{l}74,60 \\
74,66 \\
74.49 \\
74.08 \\
75,02 \\
74.66 \\
74.22 \\
74.21 \\
74.93 \\
74.76\end{array}$ & $\begin{array}{l}0.22 \\
0.16 \\
0.16 \\
0,19 \\
0.17 \\
0.21 \\
0.19 \\
0.22 \\
0,16 \\
0.28\end{array}$ & $\begin{array}{l}11,64 \\
11,67 \\
11,46 \\
11,69 \\
11,14 \\
11,59 \\
11,31 \\
11,66 \\
11,71 \\
11,40\end{array}$ & $\begin{array}{l}2,14 \\
2,14 \\
2,11 \\
2.08 \\
2,40 \\
2,09 \\
3,03 \\
2.03 \\
2,16 \\
3,06\end{array}$ & $\begin{array}{l}0.08 \\
0.10 \\
0.10 \\
0.05 \\
0.08 \\
0.09 \\
0.05 \\
0.12 \\
0.05 \\
0.11\end{array}$ & $\begin{array}{l}0.09 \\
0,11 \\
0,07 \\
0.11 \\
0,02 \\
0,10 \\
0,02 \\
0.13 \\
0.11 \\
0.01\end{array}$ & $\begin{array}{l}0,84 \\
0,85 \\
0,86 \\
0,94 \\
0.86 \\
0.87 \\
1,25 \\
0,88 \\
0.94 \\
1,19\end{array}$ & $\begin{array}{l}3,25 \\
3,20 \\
3,04 \\
2,77 \\
2,79 \\
3,17 \\
2,90 \\
3,21 \\
3,23 \\
3,08\end{array}$ & $\begin{array}{l}2,68 \\
2,61 \\
2,80 \\
3,29 \\
2,83 \\
2,71 \\
2,32 \\
2,70 \\
2,61 \\
2,37\end{array}$ & $\begin{array}{l}0.05 \\
0.03 \\
0,03 \\
0,01 \\
0,01 \\
0,02 \\
0,05 \\
0.02 \\
0,01 \\
0.05\end{array}$ & $\begin{array}{l}95,59 \\
95,53 \\
95,12 \\
95,21 \\
95,32 \\
95,51 \\
95,34 \\
95,18 \\
95,91 \\
96,31\end{array}$ \\
\hline $\begin{array}{l}\text { Average } \\
\text { Minimum } \\
\text { Maximum }\end{array}$ & $\begin{array}{l}74,56 \\
74,08 \\
75,02\end{array}$ & $\begin{array}{l}0,20 \\
0,16 \\
0,28\end{array}$ & $\begin{array}{l}11,53 \\
11,14 \\
11,71\end{array}$ & $\begin{array}{l}2,32 \\
2,03 \\
3,06\end{array}$ & $\begin{array}{l}0.08 \\
0.05 \\
0.12\end{array}$ & $\begin{array}{l}0,08 \\
0,01 \\
0,13\end{array}$ & $\begin{array}{l}0,95 \\
0,84 \\
1.25\end{array}$ & $\begin{array}{l}3.06 \\
2.77 \\
3.25\end{array}$ & $\begin{array}{l}2,69 \\
2,32 \\
3,29\end{array}$ & $\begin{array}{l}0.03 \\
0.01 \\
0.05\end{array}$ & $\begin{array}{l}95,50 \\
95,12 \\
96,31\end{array}$ \\
\hline $642 C-10-6,5-7$ & $\begin{array}{l}48,24 \\
48,54 \\
48,35 \\
48,48 \\
48,20 \\
48,34 \\
48,54\end{array}$ & $\begin{array}{l}2.66 \\
2.47 \\
2.52 \\
2.69 \\
2.39 \\
2.55 \\
2.58\end{array}$ & $\begin{array}{l}13,21 \\
13,58 \\
13,09 \\
13,19 \\
13,29 \\
13,19 \\
13,25\end{array}$ & $\begin{array}{l}12,66 \\
13,42 \\
13,21 \\
13,07 \\
13,00 \\
12,62 \\
13,45\end{array}$ & $\begin{array}{l}0.16 \\
0.18 \\
0.09 \\
0.16 \\
0.15 \\
0.25 \\
0.25\end{array}$ & $\begin{array}{l}6,38 \\
6,59 \\
6,16 \\
6,46 \\
6,63 \\
6,51 \\
6,07\end{array}$ & $\begin{array}{l}12,13 \\
12,05 \\
12,09 \\
12,26 \\
12,19 \\
12,50 \\
11,97\end{array}$ & $\begin{array}{l}2,40 \\
2,52 \\
2,51 \\
2,60 \\
2,57 \\
2,47 \\
2,72\end{array}$ & $\begin{array}{l}0.27 \\
0.28 \\
0.34 \\
0.29 \\
0.29 \\
0.28 \\
0.27\end{array}$ & $\begin{array}{l}0.27 \\
0.21 \\
0.39 \\
0.19 \\
0.20 \\
0.23 \\
0.22\end{array}$ & $\begin{array}{l}98,38 \\
99,84 \\
98,75 \\
99,39 \\
98,91 \\
98,94 \\
99,32\end{array}$ \\
\hline $\begin{array}{l}\text { Average } \\
\text { Minimum } \\
\text { Maximum }\end{array}$ & $\begin{array}{l}48,38 \\
48,20 \\
48,54\end{array}$ & $\begin{array}{l}2,55 \\
2,39 \\
2,69\end{array}$ & $\begin{array}{l}13,26 \\
13,09 \\
13,58\end{array}$ & $\begin{array}{l}13,06 \\
12,62 \\
13,45\end{array}$ & $\begin{array}{l}0.18 \\
0.09 \\
0.25\end{array}$ & $\begin{array}{l}6,40 \\
6,07 \\
6,63\end{array}$ & $\begin{array}{l}12,17 \\
11,97 \\
12,50\end{array}$ & $\begin{array}{l}2,54 \\
2,40 \\
2,60\end{array}$ & $\begin{array}{l}0,29 \\
0,27 \\
0,34\end{array}$ & $\begin{array}{l}0.24 \\
0.19 \\
0.39\end{array}$ & $\begin{array}{l}99,08 \\
98,38 \\
99,84\end{array}$ \\
\hline $642 C-10-6,5-7$ & 75,59 & 0.20 & 11,99 & 2,74 & 0.11 & 0,07 & 1,11 & 1,72 & 1.98 & 0,02 & 95,53 \\
\hline $643 \lambda-7-3,66-67$ & $\begin{array}{l}48,28 \\
49,65 \\
49,34 \\
49,06 \\
49,67\end{array}$ & $\begin{array}{l}3.82 \\
4,21 \\
3,95 \\
4.04 \\
3,39\end{array}$ & $\begin{array}{l}13,89 \\
12,43 \\
12,30 \\
12,50 \\
12,86\end{array}$ & $\begin{array}{l}15,37 \\
14,17 \\
14,57 \\
15,18 \\
13,90\end{array}$ & $\begin{array}{l}0.27 \\
0.26 \\
0.24 \\
0.23 \\
0.24\end{array}$ & $\begin{array}{l}5.19 \\
4.68 \\
4.83 \\
4.78 \\
4.98\end{array}$ & $\begin{array}{r}9.83 \\
9.80 \\
9.70 \\
9.76 \\
10.03\end{array}$ & $\begin{array}{l}2,81 \\
2,77 \\
2,82 \\
2,67 \\
2,70\end{array}$ & $\begin{array}{l}0,60 \\
0,67 \\
0,62 \\
0,72 \\
0,64\end{array}$ & $\begin{array}{l}0.51 \\
0.45 \\
0.41 \\
0.50 \\
0.37\end{array}$ & $\begin{array}{r}100,57 \\
99,09 \\
98,78 \\
99,44 \\
98,78\end{array}$ \\
\hline $\begin{array}{l}\text { Average } \\
\text { Minimum } \\
\text { Maximum }\end{array}$ & $\begin{array}{l}49,20 \\
48,28 \\
49,67\end{array}$ & $\begin{array}{l}3,88 \\
3,39 \\
4.21\end{array}$ & $\begin{array}{l}12,80 \\
12,30 \\
13,89\end{array}$ & $\begin{array}{l}14,64 \\
13,90 \\
15,37\end{array}$ & $\begin{array}{l}0.25 \\
0.23 \\
0.27\end{array}$ & $\begin{array}{l}4,89 \\
4,68 \\
5,19\end{array}$ & $\begin{array}{r}9,82 \\
9,70 \\
10.03\end{array}$ & $\begin{array}{l}2,75 \\
2,67 \\
2,82\end{array}$ & $\begin{array}{l}0,65 \\
0,60 \\
0.72\end{array}$ & $\begin{array}{l}0,45 \\
0,37 \\
0,51\end{array}$ & $\begin{array}{r}99,33 \\
98,78 \\
100,57\end{array}$ \\
\hline $643 \lambda-7-3,66-67$ & $\begin{array}{l}76,48 \\
73,69 \\
75,10 \\
76,83 \\
76,56 \\
76,90\end{array}$ & $\begin{array}{l}0,44 \\
0,79 \\
0,58 \\
0,25 \\
0,22 \\
0.21\end{array}$ & $\begin{array}{l}12,36 \\
12,71 \\
12,16 \\
11,89 \\
12,02 \\
12,14\end{array}$ & $\begin{array}{l}2,42 \\
3,60 \\
2,65 \\
2,44 \\
2,48 \\
2,61\end{array}$ & $\begin{array}{l}0,15 \\
0,13 \\
0,12 \\
0,14 \\
0,18 \\
0,10\end{array}$ & $\begin{array}{l}0,19 \\
0,53 \\
0,28 \\
0,10 \\
0,13 \\
0,08\end{array}$ & $\begin{array}{l}1,07 \\
2.13 \\
1,59 \\
1,09 \\
1.09 \\
1,09\end{array}$ & $\begin{array}{l}1,30 \\
1,20 \\
1,64 \\
1,90 \\
1,83 \\
2.22\end{array}$ & $\begin{array}{l}1,81 \\
1,44 \\
1,71 \\
2,15 \\
2,18 \\
2,14\end{array}$ & $\begin{array}{l}0,08 \\
0,03 \\
0,30 \\
0,00 \\
0,10 \\
0,04\end{array}$ & $\begin{array}{l}96,30 \\
96,25 \\
96,13 \\
96,79 \\
96,79 \\
97,53\end{array}$ \\
\hline $\begin{array}{l}\text { Average } \\
\text { Minimum } \\
\text { Maximum }\end{array}$ & $\begin{array}{l}75,93 \\
73,69 \\
76,90\end{array}$ & $\begin{array}{l}0,42 \\
0,21 \\
0,79\end{array}$ & $\begin{array}{l}12,21 \\
11,89 \\
12,71\end{array}$ & $\begin{array}{l}2,70 \\
2,42 \\
3,60\end{array}$ & $\begin{array}{l}0,14 \\
0,10 \\
0,18\end{array}$ & $\begin{array}{l}0.22 \\
0.08 \\
0.53\end{array}$ & $\begin{array}{l}1.34 \\
1.07 \\
2.13\end{array}$ & $\begin{array}{l}1,68 \\
1,20 \\
2,22\end{array}$ & $\begin{array}{l}1,91 \\
1,44 \\
2,18\end{array}$ & $\begin{array}{l}0,09 \\
0,00 \\
0,30\end{array}$ & $\begin{array}{l}96,63 \\
96,13 \\
97,53\end{array}$ \\
\hline $643 \lambda-2-3,74-75$ & $\begin{array}{l}50,13 \\
50,04 \\
49,63 \\
48,62\end{array}$ & $\begin{array}{l}3,23 \\
3,28 \\
3,36 \\
3,43\end{array}$ & $\begin{array}{l}12,51 \\
12,63 \\
12,64 \\
12,58\end{array}$ & $\begin{array}{l}13,06 \\
13,46 \\
13,73 \\
14,03\end{array}$ & $\begin{array}{l}0.23 \\
0.20 \\
0.30 \\
0.27\end{array}$ & $\begin{array}{l}4.15 \\
4.19 \\
4.30 \\
4.34\end{array}$ & $\begin{array}{l}8,92 \\
8,86 \\
8,90 \\
9,25\end{array}$ & $\begin{array}{l}3,02 \\
3,13 \\
3,10 \\
3,04\end{array}$ & $\begin{array}{l}0,83 \\
0,79 \\
0,83 \\
0,75\end{array}$ & $\begin{array}{l}\text { n.d. } \\
\text { n.d. } \\
\text { n.d. } \\
\text { n.d. }\end{array}$ & $\begin{array}{l}96,08 \\
96,58 \\
96,79 \\
96,31\end{array}$ \\
\hline $\begin{array}{l}\text { Average } \\
\text { Minimum } \\
\text { Maximum }\end{array}$ & $\begin{array}{l}49.61 \\
48.62 \\
50,13\end{array}$ & $\begin{array}{l}3.33 \\
3.23 \\
3.43\end{array}$ & $\begin{array}{l}12,59 \\
12,51 \\
12,64\end{array}$ & $\begin{array}{l}13.57 \\
13,06 \\
14.03\end{array}$ & $\begin{array}{l}0.25 \\
0.20 \\
0.30\end{array}$ & $\begin{array}{l}4.25 \\
4.15 \\
4.34\end{array}$ & $\begin{array}{l}8.98 \\
8.86 \\
9.25\end{array}$ & $\begin{array}{l}3,07 \\
3,02 \\
3,13\end{array}$ & $\begin{array}{l}0,80 \\
0,75 \\
0,83\end{array}$ & $\begin{array}{l}\text { n.d. } \\
\text { n.d. } \\
\text { n.d. }\end{array}$ & $\begin{array}{l}96.44 \\
96.08 \\
96,79\end{array}$ \\
\hline
\end{tabular}

terpreted as being formed by hydrovolcanic/phreatomagmatic eruption mechanisms.

Colorless, rhyolitic pumice shards are larger than brown, icelanditic to tholeiitic shards and typically exceed phenocrysts in size. These size patterns might be explained by two processes.
First, highly vesicular, low-density rhyolitic pumice is erupted to greater height than basaltic glass and can thus be transported farther downwind. Second, during eolian sedimentation, dense nonvesicular lithics, crystals, and basaltic glass fragments are deposited closer to the source than less-dense rhyolitic pumice. 

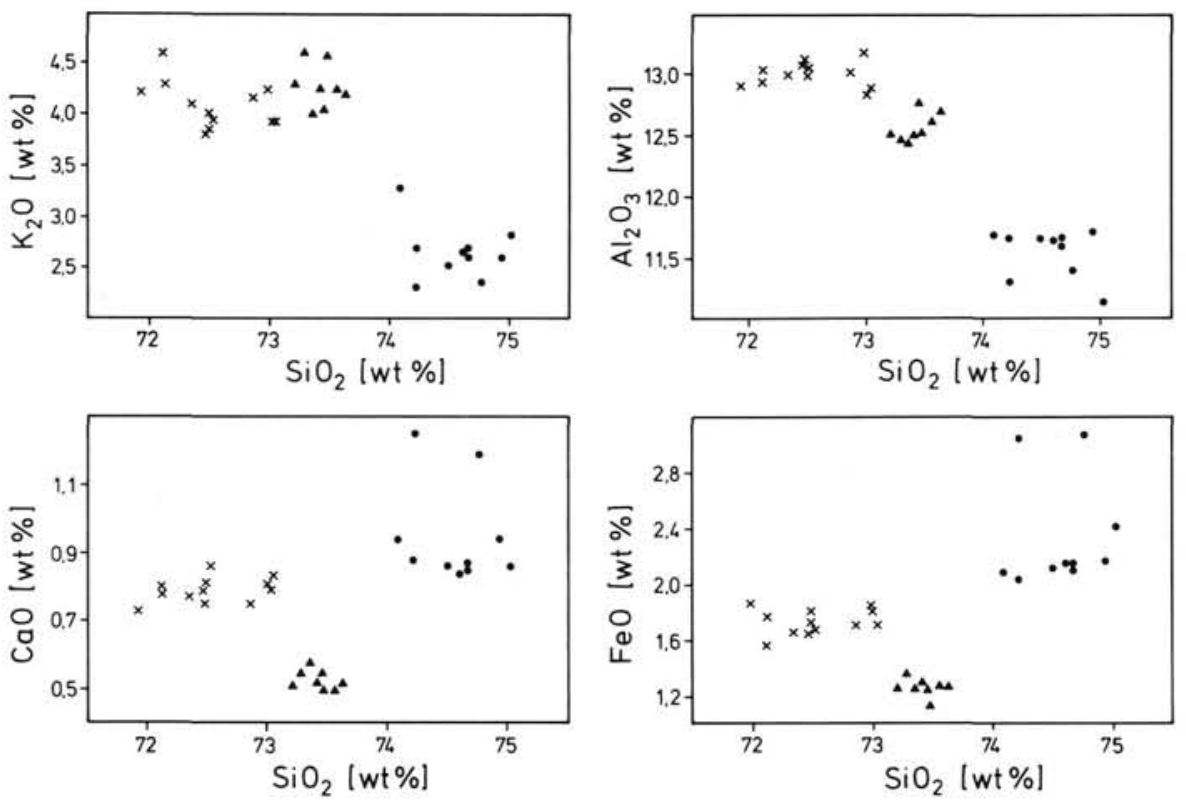

Figure 5. Major-element variation diagrams for rhyolitic glass from Samples 104-642B-13-CC, 9$13 \mathrm{~cm}(\mathrm{x}), 104-642 \mathrm{C}-15-2,110-114 \mathrm{~cm}$ (filled circles) and 104-643A-11-1, 104-105 cm (filled triangles).

Table 3. Microprobe analyses of single plagioclases from Holes 642B and 643A.

\begin{tabular}{|c|c|c|c|c|c|c|c|c|c|c|}
\hline Sample & $\mathrm{SiO} 2$ & A1203 & $\mathrm{FeO}$ & $\mathrm{CaO}$ & $\mathrm{K} 20$ & $\mathrm{Na} 2 \mathrm{O}$ & Sum & $\mathrm{Ab}$ & or & An \\
\hline $642 B-13-C C, 9-13$ & 56,10 & 26,48 & 0,47 & 9,99 & 0,35 & 5,64 & 99,03 & 49,51 & 2,02 & 48,47 \\
\hline $643 A-11-1, \quad 104-10$ & 62,02 & 22,96 & 0,31 & 5,38 & 0,33 & 8,28 & 99,28 & 72,19 & 1,89 & 25,91 \\
\hline
\end{tabular}

Eruption from zoned magma columns is suggested by the bimodal ash compositions, for instance in layers 104-642C-10-6, $5-7 \mathrm{~cm}$, and 104-643A-7-3, 66-67 cm, and by the occurrence of slight chemical and color zonation of thick rhyolitic ash layers, although we cannot exclude the less likely simultaneous eruptions from different but nearby sources.

\section{Correlation and Sources}

Correlation of the prominent layers from Sites 642 and 643 help establish a regional stratigraphy and may provide tiepoints for calibration of the Neogene time scale. Individual Pliocene to Pleistocene ash layers can be correlated based on petrographic analysis (median and maximum grain size, shape, and color of glass, mineral assemblages) and stratigraphic position as suggested by Eldholm, Thiede, Taylor, et al. (1987):

Correlation of individual Pliocene and Pleistocene ash layers

Sample from Site 642

Sample from Site 643

$104-642 \mathrm{C}-2-4,88-98 \mathrm{~cm}$ $104-642 \mathrm{C}-5-3,92-94 \mathrm{~cm}$ $104-642 \mathrm{C}-7-1,133-140 \mathrm{~cm}$ 104-642B-9-3, 96-98 cm

Correlation of single Neogene ash layers, as based on shipboard data (Eldholm, Thiede, Taylor, et al., 1987), was tested by geochemical means. Individual upper Miocene rhyolitic ash layers cannot be correlated between drill sites, because the compositions of all shards and plagioclases analyzed differ signifi- cantly between these layers (Tables 2 and 3; Fig. 5). Layers 104642C-10-6, 5-7 cm and 104-643A-7-3, 66-67 cm have bimodal compositions (Table 2; Fig. 6), have the same maximum grain size $(280 \mu \mathrm{m})$, and show similar proportions of colorless to brown glass shards (Fig. 4), but the glass compositions again differ significantly. The proposed correlation between these layers (Eldholm, Thiede, Taylor, et al., 1987) is thus not tenable, even though the ash layers analyzed have close magmatic relationships and some may have originated from very similar sources.

The petrographic similarity and abundance of ash layers in the upper Miocene intervals between 113 to 137 mbsf (Site 642) and 90 to 110 mbsf (Site 643), however, suggests that these could be correlated as a group (Fig. 2) even though individual layers cannot yet be matched between sites. Correlation of layers older than late Miocene is difficult for several reasons: disturbed sedimentary record with some hiatuses; no unique mineral assemblages in single layers; insufficient geochemical data.

Miocene to Holocene ash layers from the North Atlantic were previously recovered on the Rockall Plateau (DSDP Leg 48, Sites 403 to 406 , and Leg 81 , Sites 552 to 555; Montadert, Roberts, et al., 1979; Roberts, Schnitker, et al., 1984), on the Reykjanes Ridge (Leg 49, Sites 407 to 409; Luyendyk, Cann, et al., 1978), on the Iceland-Faeroe Ridge, the Iceland Plateau, the Jan Mayen Ridge, the Vøring Plateau, and the Norway Basin (Leg 38, Sites 336 to 352; Talwani, Udintsev, et al., 1976). The ages, ash layer thicknesses, grain sizes, and shard and mineral compositions of these Neogene ashes are all generally similar to the Vøring Plateau ashes described here (Ruddiman and Glover, 1972; Talwani and Eldholm, 1972, 1977; Talwani, Udintsev et al., 1976; Sylvester, 1978; Varet and Metrich, 1978; Donn 

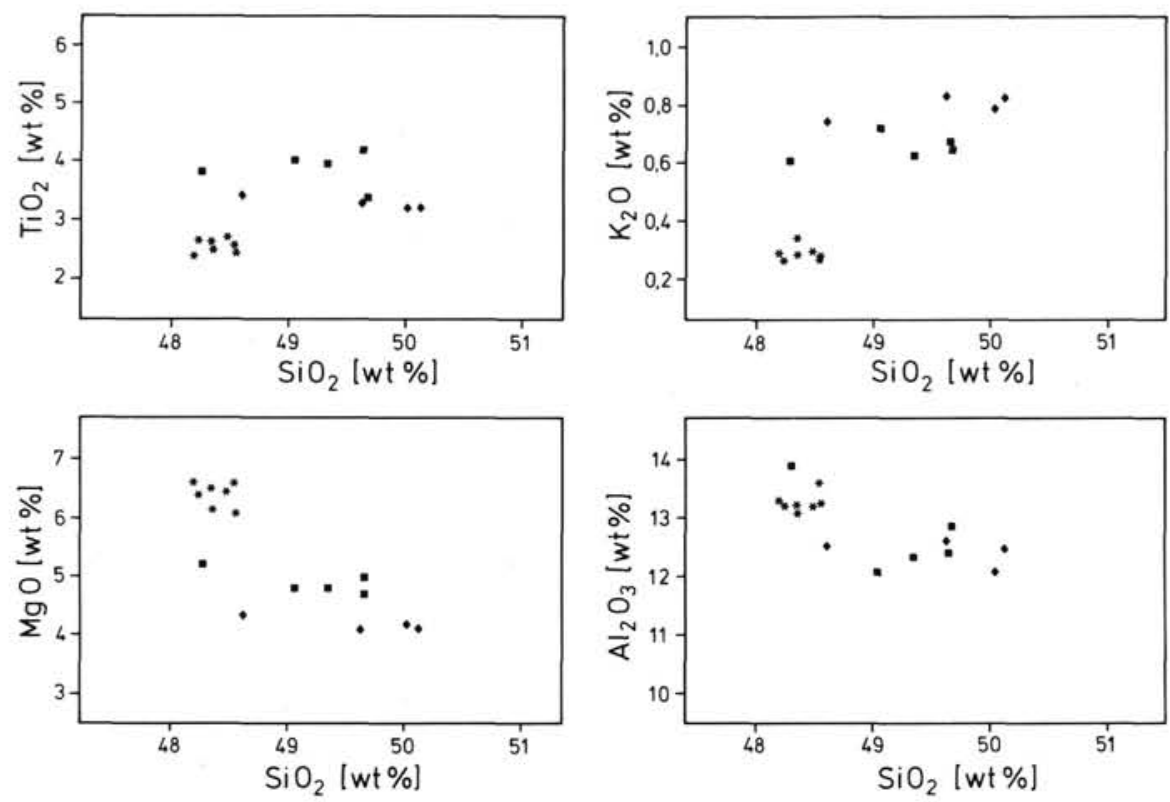

Figure 6. Major-element variation diagrams for basaltic glass from Samples 104-642C-10-6, 5-7 $\mathrm{cm}$ (stars), 104-643A-2-3, 74-75 cm (filled quadrangles) and 104-643A-7-3, 66-67 cm (filled rhombs).

and Ninkovich, 1980; Sigurdsson and Loebner, 1981). Single ash layers, however, have yet to be correlated throughout the North Atlantic Ocean Basin.

Possible ash sources lie somewhere to the south and west of the Vøring Plateau. The volcanic islands of Iceland and Jan Mayen, the Jan Mayen Ridge, and continental Greenland (even if Neogene volcanism is not yet reported there) are the most likely source areas, as they have well-documented records of $\mathrm{Ce}$ nozoic volcanic activity. Iceland is a likely source area because of its proximity, basaltic to rhyolitic magmatism, and common explosive eruptions (e.g., Sigurdsson and Loebner, 1981; Schmincke et al., 1982). Median grain sizes of about $100 \mu \mathrm{m}$, average layer thicknesses of 1-4 cm, basaltic and rhyolitic compositions of the Neogene tephra, and prevailing wind directions (Donn and Ninkovich, 1980) also suggest Iceland as the likely source area for most ashes. Historical eruptions and the distribution of volcanic ash in surface sediments show that stratospheric transport is dominantly to the northeast and east of Iceland (Sigurdsson and Loebner, 1981).

Until now, only Quaternary K-rich rhyolitic rocks have been reported from Iceland (Sigurdsson and Loebner, 1981; Schmincke et al., 1982). Both high-K and low-K compositional series are present in the ash layers from the Vøring Plateau. Sigurdsson and Loebner (1981) distinguished high-K and low-K rhyolites among Leg 38 ash layers from the North Atlantic (Fig. 7, Sites 338 and 342), both associated with rifting and opening of the Norwegian and Greenland Seas. They inferred igneous activity on the East Greenland continental margin or near the Iceland-Faeroe Ridge as possible sources for middle Oligocene high-K silicic ashes. Only Eocene to early Miocene high-K ashes can be attributed to these provinces, because the youngest east Greenland igneous activity with high-K rhyolites is early Miocene in age (Gleadow et al., 1979) and volcanic activity on the Faeroe Islands ceased until middle Miocene. High-K magmatism from Jan Mayen Island, on the other hand, is at least as young as Pliocene-Pleistocene (Fitch et al., 1965; Sylvester, 1975; Maaloe et al., 1986). Thus, late Miocene K-rich rhyolitic ashes described here cannot have originated in either Jan Mayen Island, Greenland, or Iceland. We suggest that the entire area encompassing the Iceland-Jan Mayen Ridge was a possible source for late Miocene K-rich silicic explosive eruptions (Fig. 7), even though individual volcanic centers are unknown so far. We speculate that during the late Miocene large rhyolitic eruptive centers in what may be called Jan Mayen magmatic province, which is characterized by K-rich alkalic magmas, were active have since foundered. The unique nature of the K-rich Jan Mayen area, when compared with other volcanic areas in the Atlantic Ocean north of the Azores, was noted previously (Schmincke,

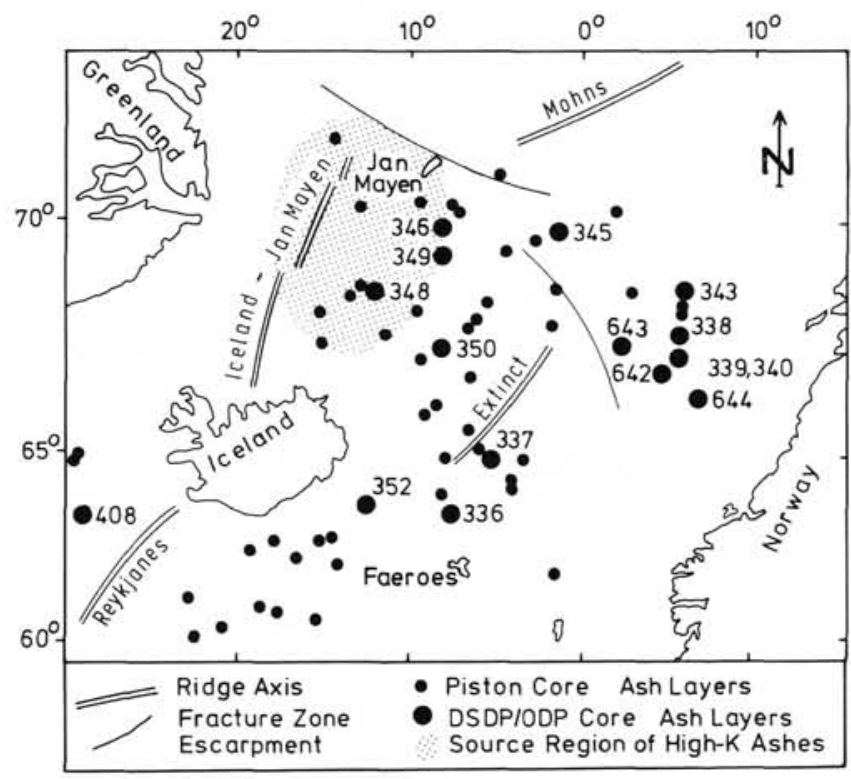

Figure 7. Map of the North Atlantic Ocean around Iceland with locations of ash-bearing piston cores and DSDP/ODP sites (completed after Donn and Ninkovich, 1980). Numbers refer to DSDP/ODP sites; seafloor structural features after Talwani and Eldholm (1977). Stipled area represents the Jan Mayen magmatic province inferred as the possible source region for Neogene high-K ashes in the North Atlantic north of Iceland. 
1973). Jan Mayen Island is a possible source for Quaternary alkali-rich layers with K-rich mineral assemblages (biotite- and feldspar-rich layer 104-642B-6-1, 132-133 cm) based on the Pliocene-Pleistocene age of its magmatism. Sylvester (1978) reported well-sorted ashes with average grain sizes between 100 and $200 \mu \mathrm{m}$ from DSDP drill sites (Leg 38) near Jan Mayen Island. He inferred sources for the ashes in Iceland and Jan Mayen Island, but also suggested separate and yet unknown sources. The basaltic ashes analyzed are very similar in their major-element concentrations to basalts from Iceland and are therefore related to sources in Iceland, although we cannot definitely rule out other, yet unknown sources.

\section{Continuous or Episodic Neogene Volcanism in the North Atlantic?}

Sylvester (1978), Donn and Ninkovich (1980), and Sigurdsson and Loebner (1981) interpreted changes in the abundance of ash layers in Neogene sediments from the North Atlantic as reflecting corresponding variations in eruption frequency and therefore magmatic productivity and, in some cases, changes in spreading rates. Leg 104 Sites 642 and 643 allow a more detailed evaluation of possible volcanic episodicity because of excellent core recovery and a rather continuous sequence of Neogene sediments with an exceptionally well-preserved ash layer record.

Donn and Ninkovich (1980) used piston-core and DSDP-core ashes to infer rates of explosive volcanism in the North Atlantic during the Cenozoic. The ashes were thought to have derived from eruptions in Iceland. They make certain reservations, however, regarding their data because of poor core recovery and serious hiatuses in the sedimentary record. After interpolation for missing data and corrections for wind effects and regional plate motions, high volcanic activity was inferred for the middle Eocene and the Pliocene (Fig. 8).

In contrast, Sigurdsson and Loebner (1981), using ash layers from Leg 38, inferred four episodes of abundant explosive volcanism: middle Eocene, middle Oligocene, early to middle Miocene, Pliocene to Pleistocene (Fig. 8). These authors also caution against overinterpreting their data because of poor core recovery during Leg 38 . For instance, their inferred late Miocene decline in explosive volcanic activity is based on very poor core recovery $(4 \%)$. On the other hand, spreading rates in the North Atlantic increased in the late Miocene (Talwani and Eldholm, 1977; Sigurdsson and Loebner, 1981) and accumulation rates of basalt in Iceland increased abruptly (Saemundsson et al., 1980) at that time, contradicting the minimum in volcanic activity suggested by Sigurdsson and Loebner (1981).

The oldest ash layers from Leg 104 sites are early Miocene and are tholeiitic to icelanditic. Basaltic ashes in the North Atlantic can form widespread layers (e.g., Pedersen et al., 1975), possibly resulting from highly explosive shallow submarine or subglacial eruptions.

In the middle Miocene sequence, up to 21 ash layers were counted. Adoption of a 3.2-m.y. hiatus, as suggested by Goll (this volume) in the upper middle Miocene section, would raise the ash layer number to about 40 and frequency to 6 to 8 layers per m.y. This is so because the hiatus covers about the same time interval with an inferred similar number of ash layers as the recovered section of the middle Miocene. During the late Miocene, up to 26 layers (5 to 6 layers per m.y.) were deposited. A 1.45-m.y. hiatus within the late Miocene at Site 642 (Goll, this volume) is counterbalanced by Site 643 , where that interval and its interbedded ash layers were recovered. A conspicuous 20-m interval (113-137 m depth at Site 642, 90-110 m depth at Site 643) in the late Miocene section is particularly rich in ash layers, indicating either a peak in volcanic activity, reduced sedimentation rates, or both. Available stratigraphic and paleomagnetic data (Goll, this volume; Bleil et al., this volume) attribute

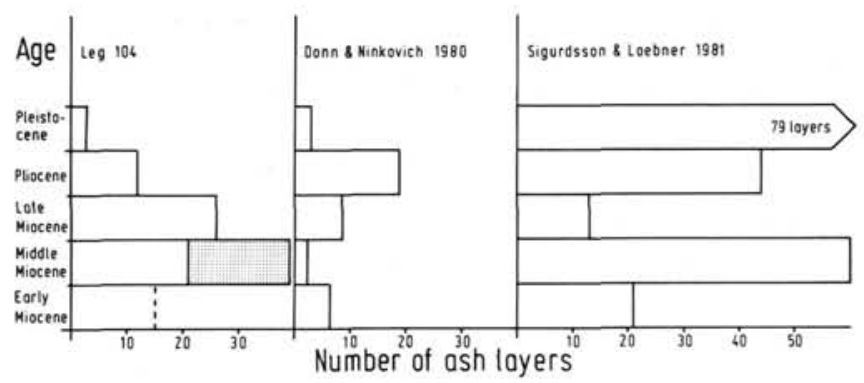

Figure 8. A comparison of the number of ash layers vs. stratigraphic age of Leg 104 cores from the Vøring Plateau, with ash layer frequencies in the North Atlantic [after Donn and Ninkovich (1980) and Sigurdsson and Loebner (1981)]. The highest number of ash layers for a given stratigraphic interval (epoch) was used for all Leg 104 cores. The stipled area represents the extrapolated, additional number of ash layers when adopting the hiatus proposed by Goll (this volume).

a duration of about 3 m.y. to this interval at Site 642, including a $1.45-\mathrm{m}$.y. hiatus. A total of 23 ash layers amounting to 7 to 8 ash layers per m.y. is calculated from this 3-m.y. section, if we adopt the number of ash layers from Site 643 for the hiatus interval. No significant peak in volcanic activity is therefore apparent. Furthermore, continuous ash layer deposition and preservation of bioturbated sediments suggest that low sedimentation rates are the reason for the high number of ash layers in the late Miocene sequence. Detailed studies of DSDP Site 341 (Leg 38) sediments revealed that, especially during the late Miocene, very low sedimentation rates prevailed on the Vøring Plateau (Smalley et al., 1986). The late Miocene is thus characterized by low sedimentation rates $(6-13 \mathrm{~mm} / \mathrm{Ka})$ but continuous volcanic ash deposition at a rate of 5 to 8 layers per m.y. Spreading rates and magmatic productivity in the North Atlantic increased at that time (Talwani and Eldholm, 1977; Saemundsson et al., 1980; Sigurdsson and Loebner, 1981).

Within the uppermost $110 \mathrm{~m}$ of Site 642 and the upper $90 \mathrm{~m}$ of Site 643, only 11 (Site 642) and 12 (Site 643) Pliocene and Holocene layers are preserved. Either volcanic activity decreased through that period or unfavorable wind and sedimentation conditions prevented ash deposition. If we combine the increase in sedimentation rate ( 16 to $50 \mathrm{~mm} / \mathrm{Ka}$ using shipboard downhole patterns in remanent magnetization polarit:' and time scale after Berggren et al., 1985; see also data from Caston et al., 1976) with the onset of glacial conditions somewhere between 2.4 Ma (Shackleton et al., 1984) and $4 \mathrm{Ma}$ (Eldholm, Thiede, Taylor, et al., 1987), a possible ice cover and ice rafting and a Pliocene hiatus (Goll, this volume) may explain low ash sedimentation on the Vøring Plateau during Pliocene to Holocene times.

The pronounced peaks in Pliocene (Donn and Ninkovich, 1980 ) or middle Miocene (Sigurdsson and Loebner, 1981) volcanic activity in the North Atlantic cannot be confirmed from the Vøring Plateau ash layers (Fig. 8). The apparent low ash layer frequency of Sigurdsson and Loebner (1981) in the late Miocene actually corresponds to vigorous volcanic activity shown by 26 ash layers in Leg 104 sites (Fig. 8).

\section{CONCLUSIONS}

1. The ash layers studied have fairly uniform median grain sizes (about $100 \mu \mathrm{m}$ ) but very different (280 to $1200 \mu \mathrm{m})$ maximum grain sizes, related to the rhyolitic glasses. Unique mineral assemblages are lacking except in one distinctive, biotite-bearing layer.

2. Bimodal geochemical compositions are indicated by rhyolitic shards in dominantly basaltic layers, whereas some brown 
shards in the rhyolitic layers are also rhyolitic. The basaltic glasses analyzed are tholeiitic and can be divided into primitive medium-Ti and more evolved high-Ti basalts. The latter cannot be derived from the medium-Ti basalts by simple fractional crystallization, but all basaltic shards are compositionally similar to basalts from Iceland. The rhyolites comprise both low-K and high-K types with distinctive feldspar phenocrysts. Except for a few Pliocene to Holocene ash layers, no correlation between previously correlated ash layers in different Leg 104 drill sites can be established.

3. Iceland is the most likely source for the basaltic and the low-K rhyolitic ashes. The K-rich Jan Mayen magmatic province is inferred as source region for late Miocene, high-K rhyolitic ashes and for Quaternary, biotite-bearing ash layers. The ashes deposited on the Vøring Plateau probably resulted from powerful Plinian eruptions and possibly some pumice rafting.

4. The particularly well-preserved record of Neogene to Holocene ash layers does not support the highly episodic volcanic activity advocated for this part of the North Atlantic (Norwegian-Greenland Sea) by previous authors.

Average calculated ash layer deposition rates are between 5 and 8 layers per m.y. for Neogene epochs, and around 3 layers per m.y. for Pliocene to Holocene times. The latter number, however, is obscured by the unknown influence of an ice cover precluding fallout sedimentation onto the ocean floor.

\section{ACKNOWLEDGMENTS}

R. Goll, U. Bleil, R. Henrich, and G. Bohrmann provided valuable stratigraphic information. Some of the polished sections were obtained through the kind help of J. P. Huijsmans. Many thanks to W. Schnetger Köhler, A. Freundt, and U. Bednarz, who assisted us with the microprobe work, and W. Lehmann, who helped with the drafting. A first version of this paper was substantially improved by the reviews of G. Heiken and S. Carey. To all of them and the Leg 104 Shipboard Scientific Party we owe thanks. Financial support by the DFG (Grant schm.250/37-1 to H.-U. Schmincke) is gratefully acknowledged.

\section{REFERENCES}

Berggren, W. A., Kent, D. V., Flynn, J. J., and Van Couvering, J. A., 1985. Cenozoic geochronology. Geol. Soc. Am. Bull., 96:1407-1418.

Caston, V.N.D., 1976. Tertiary sediments of the Vøring Plateau, Norwegian Sea, recovered by Leg 38 of the Deep Sea Drilling Project. In Talwani, M., Udintsev, G., et al., Init. Repts. DSDP, 38: Washington (U.S. Govt. Printing Office), 761-782.

Donn, W. L., and Ninkovich, D., 1980. Rate of Cenozoic explosive volcanism in the North Atlantic Ocean inferred from deep sea cores. $J$. Geophys. Res., 85:5455-5460.

Eldholm, O., Thiede, J., Taylor, E., et al., 1987. Proc. ODP, Init. Repts., 104: College Station (Ocean Drilling Program).

Fisher, R. V., and Schmincke, H.-U., 1984. Pyroclastic Rocks: New York (Springer-Verlag).

Fitch, F. J., Grasty, D. L., and Miller, J. A., 1965. Potassium-argon ages of rocks from Jan Mayen and an outline of its volcanic history. Nature, 207:1349-1351.

Gleadow, A.J.W., and Brooks, C. K., 1979. Fission track dating, thermal histories and tectonics of igneous intrusions in east Greenland. Contrib. Mineral. Petrol., 71:45-60.

Flower, M.F.J., Pritchard, R. G., Brem, G., Cann, J. R., Delaney, J., Emmermann, R., Gibson, I. L., Oakley, P. J., Robinson, P. T., and
Schmincke, H.-U., 1982. Chemical stratigraphy, Iceland research drilling project Reydarfjørdur, eastern Iceland. J. Geophys. Res., 87: 6489-6510.

Jorgensen, K. A., 1980. The Thorsmörk ignimbrite: An unusual comenditic pyroclastic flow in southern Iceland. J. Volcanol. Geotherm. Res., 8:7-22.

Luyendyk, B. P., Cann, J. R., et al., 1978. Init. Repts. DSDP, 49: Washington (U.S. Govt. Printing Office).

Maaloe, S., Sørensen, I., and Hertogen, J., 1986. The trachybasaltic suite of Jan Mayen. J. Petrol., 27:439-466.

Montadert, L., Roberts, D. G., et al., 1979. Init. Repts. DSDP, 48: Washington (U.S. Govt. Printing Office).

Mutter, J. C., Talwani, M., and Stoffa, P. L., 1984. Evidence for a thick oceanic crust adjacent to the Norwegian margin. J. Geophys. Res., 89:483-502.

Pedersen, A. K., Engell, J., and Ronsbø, J. G., 1975. Early Tertiary volcanism in the Skagerrak: new chemical evidence from ash-layers in the Moclay of northern Denmark. Lithos, 8:255-268.

Roberts, D. G., Schnitker, D., et al., 1984. Init. Repts. DSDP, 81: Washington (U.S. Govt. Printing Office).

Ruddiman, W. F., and Glover, L. K., 1972. Vertical mixing of ice-rafted volcanic-ash in North Atlantic sediments. Geol. Soc. Am. Bull., 83: 2817-2836.

Saemundsson, K., Kristjansson, L., McDougall, I., and Watkins, N. D., 1980. K-Ar dating, geological and paleomagnetic study of a $5-\mathrm{km}$ lava succession in Northern Iceland. J. Geophys. Res., 85:3628-3646.

Schmincke, H.-U., 1973. Magmatic evolution and tectonic regime in the Canary, Madeira, and Azores island groups. Geol. Soc. Am. Bull., 84:633-648.

Schmincke, H.-U., Viereck, L., Griffin, B. J., and Pritchard, G., 1982. Volcaniclastic rocks of the Reydarfjørdur drill hole, eastern Iceland 1. Primary features. J. Geophys. Res., 87:6437-6458.

Shackleton, N. J., Backman, J., et al., 1984. Oxygen isotope calibration of the onset of ice-rafting and history of glaciation in the North Atlantic region. Nature, 307:620-623.

Sigurdsson, H., and Loebner, B., 1981. Deep-sea record of Cenozoic explosive volcanism in the North Atlantic. In Self, S., and Sparks, R. S. J. (Eds.), Tephra Studies: Dordrecht, Netherlands (Reidel Publications), 289-316.

Smalley, P. C., Nordaa, A., and Råheim, A., 1986. Geochronology and paleothermometry of Neogene sediments from the Vøring Plateau using Sr, C and O isotopes. Earth Planet. Sci. Lett., 78:368-378.

Sylvester, A. G., 1975. History and surveillance of volcanic activity on Jan Mayen Island. Bull. Volcanol., 39/2:1-23.

Sylvester, A. G., 1978. Petrography of volcanic ashes in deep-sea cores near Jan Mayen Island: Sites 338, 345-350 DSDP Leg 38. In Talwani, M., Udintsev., G., et al., Init. Repts. DSDP, Suppl. to 38, 39, 40, 41: Washington (U.S. Govt. Printing Office), 101-109.

Talwani, M., and Eldholm, O., 1972. The continental margin off Norway. A geophysical study. Geol. Soc. Am. Bull., 83:3575-3608.

1977. Evolution of the Norwegian-Greenland Sea. Geol. Soc. Am. Bull., 88:969-999.

Talwani, M., Udintsev, G., et al., 1976. Init. Repts. DSDP, 38: Washington (U.S. Govt. Printing Office).

Varet, J., and Metrich, N., 1978. Ash layers interlayered with the sediments of Holes 407 and 408, IPOD Leg 49. In Luyendyk, B. P., Cann, J. R., et al., Init. Repts. DSDP, 49: Washington (U.S. Govt. Printing Office), 437-441.

Walker, G.P.L., 1966. Acid volcanic rocks in Iceland. Bull. Volcanol., 29:375-406.

Date of initial receipt: 29 January 1988

Date of acceptance: 4 August 1988

Ms 104B-129 